\title{
Front Matter: Volume 11333
}

, "Front Matter: Volume 11333," Proc. SPIE 11333, AOPC 2019: Advanced Laser Materials and Laser Technology, 1133301 (18 December 2019); doi: $10.1117 / 12.2563994$

SPIE. Event: Applied Optics and Photonics China (AOPC2019), 2019, Beijing, China 


\title{
PROCEEDINGS OF SPIE
}

\section{AOPC 2019: Advanced Laser Materials and Laser Technology}

\author{
Pu Zhou \\ Jian Zhang \\ Wenxue Li \\ Shibin Jiang \\ Takunori Taira \\ Editors
}

7-9 July 2019

Beijing, China

Sponsored by

Chinese Society for Optical Engineering (China)

Cosponsored by

SPIE

Organized by

Chinese Society for Optical Engineering (China)

National University of Defense Technology (China)

East China Normal University (China)

Jiangsu Normal University (China)

Infrared and Laser Engineering (China)

Published by

SPIE 
The papers in this volume were part of the technical conference cited on the cover and title page. Papers were selected and subject to review by the editors and conference program committee. Some conference presentations may not be available for publication. Additional papers and presentation recordings may be available online in the SPIE Digital Library at SPIEDigitalLibrary.org.

The papers reflect the work and thoughts of the authors and are published herein as submitted. The publisher is not responsible for the validity of the information or for any outcomes resulting from reliance thereon.

Please use the following format to cite material from these proceedings:

Author(s), "Title of Paper," in AOPC 2019: Advanced Laser Materials and Laser Technology, edited by Pu Zhou, Jian Zhang, Wenxue Li, Shibin Jiang, Takunori Taira, Proceedings of SPIE Vol. 11333 (SPIE, Bellingham, WA, 2019) Seven-digit Article CID Number.

ISSN: 0277-786X

ISSN: 1996-756X (electronic)

ISBN: 9781510634381

ISBN: 9781510634398 (electronic)

Published by

SPIE

P.O. Box 10, Bellingham, Washington 98227-0010 USA

Telephone +1 3606763290 (Pacific Time) · Fax + 13606471445

SPIE.org

Copyright @ 2019, Society of Photo-Optical Instrumentation Engineers.

Copying of material in this book for internal or personal use, or for the internal or personal use of specific clients, beyond the fair use provisions granted by the U.S. Copyright Law is authorized by SPIE subject to payment of copying fees. The Transactional Reporting Service base fee for this volume is $\$ 21.00$ per article (or portion thereof), which should be paid directly to the Copyright Clearance Center (CCC), 222 Rosewood Drive, Danvers, MA 01923. Payment may also be made electronically through CCC Online at copyright.com. Other copying for republication, resale, advertising or promotion, or any form of systematic or multiple reproduction of any material in this book is prohibited except with permission in writing from the publisher. The CCC fee code is 0277$786 \times / 19 / \$ 21.00$.

Printed in the United States of America by Curran Associates, Inc., under license from SPIE.

Publication of record for individual papers is online in the SPIE Digital Library.

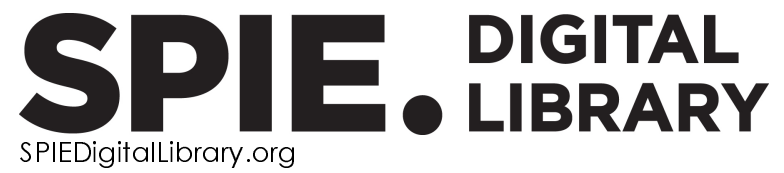

Paper Numbering: Proceedings of SPIE follow an e-First publication model. A unique citation identifier (CID) number is assigned to each article at the time of publication. Utilization of CIDs allows articles to be fully citable as soon as they are published online, and connects the same identifier to all online and print versions of the publication. SPIE uses a seven-digit CID article numbering system structured as follows:

- The first five digits correspond to the SPIE volume number.

- The last two digits indicate publication order within the volume using a Base 36 numbering system employing both numerals and letters. These two-number sets start with $00,01,02,03,04$, 05, 06, 07, 08, 09, OA, OB ... 0Z, followed by 10-1Z, 20-2Z, etc. The CID Number appears on each page of the manuscript. 


\title{
Contents
}

\author{
vii Authors \\ ix Conference Committees \\ xi Introduction
}

\section{ADVANCED LASER MATERIALS AND LASER TECHNOLOGY}

$1133302 \quad$ Experimental and theoretical research of quasi-three level $\mathrm{Ho}^{3+}$-doped fluorotellurite photonic crystal fiber lasers [1 1333-2]

$1133306 \quad 6.2 \mathrm{~kW}$ spectral beam combination by means of edge filters [11333-6]

1133307 A type of lidar sensor uses a $1645 \mathbf{~ n m ~ l a s e r ~ u s i n g ~ T i S 2 ~ a s ~ a ~ s a t u r a b l e ~ a b s o r b e r ~ [ 1 1 3 3 3 - 7 ] ~}$

1133308 Direct AIGaAs LD pumped 2.1 $\mathrm{\mu m}$ Tm/Ho composite laser [11333-9]

$1133309 \quad$ Few-layer NiPS3 as a saturable absorber for pulse laser generation [1 1333-11]

$113330 \mathrm{~A}$ Athermalisation design and analysis of the opto-mechanical structure based on laser radar launching system [11333-12]

$11333 \mathrm{OB}$ The investigation of the ramping length of the stainless steel railway vehicle laser welding seam [11333-13]

11333 OC Polarization properties of a stochastic electromagnetic ultrashort pulse laser beam in the atmospheric turbulence [11333-15]

11333 OD High efficiency slab Tm:YAP laser dual-end-pumped by fiber coupled laser diodes [11333-16]

$11333 \mathrm{OE} \quad$ Surface modification and infrared-radar stealth performance of of $\mathrm{La}_{0.7} \mathrm{Ba}_{0.3} \mathrm{MnO}_{3} \mathrm{powder}$ [11333-17]

11333 OF L-band tunable and switchable multi-wavelength erbium-doped fiber laser [11333-18]

$113330 \mathrm{D}$ Discussion and implementation of LDPC and PPM joint coding in laser underwater data transmission [11333-21]

$11333 \mathrm{OH} \quad$ First principles study electronic and optical properties of $\mathrm{LaBr}_{3}$ and $\mathrm{LaBr}_{3}: \mathrm{Ce}$ crystal [1 1333-22]

$11333 \mathrm{Ol} \quad$ The characteristic of wedge waves propagating along wedge with periodic V-defects by laser ultrasound technique [11333-23] 
$113330 \mathrm{~J} \quad$ Realization of 7-channel beam coherent beam combination based on coherent fiber-opticsarray collimator [11333-24]

11333 OK Intensity distribution of vortex beams from multilayered topological insulator slab [1 1333-25]

$11333 \mathrm{OL} \quad$ Effect of spot size on laser damage single crystal silicon [11333-27]

11333 OM LD end-pumped 12J-10Hz Nd:YAG pulse laser [11333-28]

1133300 Research on design of laser pyrotechnic system for high temperature-resistant transmission path [11333-30]

11333 OP Research on the application of laser pyrotechnic device to airborne escape system [1 1333-31]

$113330 Q \quad$ Evolution properties of array laser beams through an atmospheric furbulence and shock-wave layer [11333-32]

$11333 \mathrm{OR} \quad$ A review of carbon-based microwave absorbing composites [11333-33]

11333 OS Summarize the damage caused by different types of laser beams [11333-34]

11333 OT An approach to effect-based laser weapon design [1 1333-35]

11333 OU Deep transfer learning for underwater vehicle wake recognition in infrared imagery [11333-36]

$113330 \mathrm{~V} \quad$ Hybrid laser-arc welding process of full penetration $\mathrm{T}$ joint in high speed railway vehicle bogie [11333-37]

11333 oW Theoretical evaluation of V-pumped thin-disk DPALs [1 1333-38]

$113330 X \quad$ Effect of lateral index step on the performance of high-power broad-area 970-nm diode lasers based a large-optical-cavity waveguide structure [11333-39]

11333 oY Control Airy-Talbot effect via initial launch angles in dynamic linear index potentials [1 1333-40]

1133310 Finite element analysis of focus tunable micro-lens based on electric field modulated liquid crystal [11333-42]

$1133311 \quad$ Point-by-point fiber gratings inscription by using high repetition rate femtosecond laser [11333-43]

$1133312 \quad$ Numerical study on flow and heat transfer of a finned-tube heat exchanger with trapezoid vortex generators in $\mathrm{CO}_{2}$ lasers [1 1333-44]

$1133314 \quad$ Vortex structures in the photoelectron momentum distributions of negative hydrogen ions induced by a short laser pulse [11333-46] 
$1133315 \quad$ Adaptive temporal shape control of fiber laser [11333-47]

$1133316 \quad$ Dynamic error modeling and analysis based on angle intersection measurement system [11333-48]

1133317 Research on fiber transceiver collimator based on optical fiber bundles [11333-49]

1133318 Optical fiber bundles converting pulsed lasers into continuous waves for improved measurement linearity in spectrophotometry [11333-50]

1133319 Coherent beam combination based on particle swarm optimization algorithm [11333-51]

11333 1A Magnetic-field-assisted femtosecond laser drilling of bone in ambient environment [1 1333-52]

11333 1B A novel denoising algorithm for photon-counting laser data based on LDBSCAN [1 1333-53]

11333 1D Research of heat dissipation structure with conical quartz tube for pump combiner [11333-56]

$11333 \mathrm{IE} \quad$ Influence of thermal treatment on tensile strength of fiber splicing point [11333-57]

$11333 \mathrm{IF} \quad$ Effect of scanning speed on microstructure and properties of $30 \mathrm{Cr} 3 \mathrm{Ni} 2$ alloy steel prepared by direct laser deposition [1 1333-59]

$113331 \mathrm{E} \quad$ Effect of laser scanning rate on microstructure and wear resistance of SLM high speed steel layer [1 1333-60]

$113331 \mathrm{H} \quad$ High temperature oxidation behavior of pre-laid laser melting 45CrNiMoY alloy steel reinforced by TA15 powder [11333-61]

$1133311 \quad$ Research for surface characteristics of aluminum mirrors made by laser additive manufacturing [11333-62]

$113331 \mathrm{~J} \quad$ Tunable and switchable multi-wavelength ytterbium-doped fiber laser based on nonlinear polarization rotation effect [1 1333-65]

$113331 \mathrm{~K} \quad$ Contrasted behavior of ripple morphology evolution on metals irradiated with temporally shaped femtosecond laser pulse [11333-67]

$113331 \mathrm{M} \quad$ Electro-optically gated in-line saturable absorbers for fibre lasers [11333-69] 
Proc. of SPIE Vol. $113331133301-6$

Downloaded From: https://www.spiedigitallibrary.org/conference-proceedings-of-spie on 26 Apr 2023 Terms of Use: https://www.spiedigitallibrary.org/terms-of-use 


\section{Authors}

Numbers in the index correspond to the last two digits of the seven-digit citation identifier (CID) article numbering system used in Proceedings of SPIE. The first five digits reflect the volume number. Base 36 numbering is employed for the last two digits and indicates the order of articles within the volume. Numbers start with 00, 01, 02, 03, 04, 05, 06, 07, 08, 09, 0A, OB...0Z, followed by 10-12, 20-2Z, etc.

An, Guofei, ow

Bian, Jintian, OS

Cao, Chunqiang, 0O, OP

Cen, Jing, 17

Chang, Hongxiang, 15, 17

Chang, Qi, 15, 19

Chen, Fan, 06

Chen, Guoliang, OF

Chen, Jianhong, 14

Chen, Jianhua, OO, OP

Chen, Jianzhong, 11

Chen, Jun-nan, 16

Chen, Suiyuan, $1 \mathrm{~F}, 1 \mathrm{G}, 1 \mathrm{H}$

Chen, Tao, 02

Chen, Tao, 16

Chen, Wuchao, OR

Chen, Xueting, if

Cui, Tong, 1F

Dai, Wanjun, OM

Deng, J., 08

Deng, Junjie, OR

Du, Weichuan, OX

Du, Yongcheng, OU

Gan, Haiyong, 18

Gao, Songxin, OX

Gao, Ze, 1K

Ge, Y., 08

Gladush, Yury, 1M

Guan, Yingchun, 1A

Guo, Ming, OL

Han, Juhong, OW

Han, Qingbang, ol

Han, Xiao, 11

Hao, Yue, OE

$\mathrm{He}$, Aifeng, 0O, OP

$\mathrm{He}$, Binggao, OA

$\mathrm{He}$, Guangzhong, $\mathrm{OB}, \mathrm{OV}$

$\mathrm{He}$, Ning, $0 \mathrm{G}$

$\mathrm{He}$, Yingwei, 18

Hou, Tianyue, 15, 19

Hu, Huayu, $O Q$

Huang, H. Z., 08

Huang, J. H., 08

Ivanenko, Aleksey, 1M

Jia, Jing, Ol

Jia, Wuming, $1 G$

Jiang, Hongyan, OG

Jiang, Xinying, $O M$

Jiang, Xueping, 0 I
Jiang, Zongfu, 09

Jiao, Jiancao, 11

Jiao, Li, $1 \mathrm{~K}$

Jiao, Ruiyun, OY

Jin, Guangyong, OD

Jing, BO, OO, OP

Jing, Wei, $O R$

Jing, Xufeng, 18

Kang, Nan, 11

Kobtsev, Sergey, $1 M$

Kokhanovskiy, Alexey, $1 \mathrm{M}$

Kovacevic, Radovan, OB

Li, Bin, OC

Li, Guoqiang, 07

Li, Guoyuan, 1B

Li, Hong, OL

Li, J. H., 08

Li, Kai, OB, OV

$\mathrm{Li}$, Min, $\mathrm{OH}$

Li, Xiaolong, 10

Li, Xiumei, OG

Li, Yan, OC

Li, Yang, OU

$\mathrm{Li}, \mathrm{Yi}, \mathrm{OX}$

Liang, Jing, $1 F, 1 G, 1 H$

Liao, Dongjun, $\mathrm{OQ}$

Lin, Niuniu, OK

Lin, W. X., 08

Lin, Yandong, 18

Liu, Bing, OY

Liv, Changsheng, 1F, 1G, 1H

Liv, Fang, $\mathrm{OH}$

Liu, Hui, 12

Liu, Jiawei, $\mathrm{OE}$

Liu, Sen, $0 Q$

Liv, Xiangliang, 18

Liu, Xiaoxu, OW

Liu, Yanxiang, OK

Liu, Yuan, OR

Liu, Zhongbo, 11

Long, Jinhu, OJ

LV, Min, OF, $1 \mathrm{~J}$

Ma, Jun, 06

Ma, Pengfei, 0J, 15, 17, 19

Ma, Yanxing, 0J, 15, 17, 19

Ma, Yue, OO, OP

Ma, Yun-liang, 1D, 1E

Ming, Gao, OC

Mkrtchyan, Aram, 1M 
Nasibulin, Albert G., 1M

Nei, Lei, 16

Nyushkov, Boris, $1 \mathrm{M}$

Ouyang, Xiaoping, $\mathrm{OH}$

Ren, Shenhe, OC, OK

Rui, Zhong, OU

Shan, Minglei, $\mathrm{Ol}$

Shang, Fanmin, $1 G$

Shen, Changyu, 18

Shi, Lijuan, OA

Shi, Xinyao, 09

$\mathrm{Si}$, Lei, OJ

$\mathrm{Si}, \mathrm{XU}, 1 \mathrm{D}, 1 \mathrm{E}$

Song, Keyu, OF, 1J

Song, Peng, 02

Song, Yang, 1A

$\mathrm{Su}$, Jingqin, $\mathrm{OM}$

Su, Juan, 07

Su, Rongtao, 0J, 15, 17, 19

Sun, Miao, $1 \mathrm{H}$

Sun, Xiangyang, OA

Sun, Xiaoquan, OS

Tang, Chun, OX

Wan, Jun, OT

Wang, Chao, 11

Wang, Chunsheng, OB, OV

Wang, Haoyu, OO, OP

Wang, Hongxiao, OB, OV

Wang, Jianjiang, OE

Wang, Jianmin, 1B

Wang, Jin, 09

Wang, Jing, OY

Wang, Lei, OW

Wang, Lei, $1 \mathrm{~K}$

Wang, Mei, 1F, 1G, 1H

Wang, Mingjun, OK

Wang, Tao, 09

Wang, Tianyu, OS

Wang, Wei, 11

Wang, Weimin, 18

Wang, Xibin, $1 \mathrm{~K}$

Wang, You, OW

Wang, Zhenguo, OM

Wang, Zhenming, 1B

Wei, Cong, 06

Wei, Mingwei, $1 \mathrm{H}$

Wen, Ya, OD

Wu, Chi, 07

Wu, Chunting, OD

Wu, Jian, 09, 0J, 17

Wu, L. X., 08

Wu, Wenlong, OM

Xia, Zhi-peng, 16

Xiao, Yike, 18

Xie, Dongping, 1B

Xie, Renwei, OT

Xiong, Zhi, 16

$X U$, Cheng-lin, 1D, 1E

Xu, Gan, 18

Xu, Yinuo, 18
Xue, Qiao, OM

Yan, Xiang, 12

Yan, Xiongwei, OM

Yang, Jiao, OW

Yang, Xin, OX

Yang, Xiongdan, 1B

Yang, Yan, OF, 1J

Yang, Zhendong, OY

Yao, Ke, OM

Yao, Xianghong, OE

Ye, Fanghong, 1B

Yuan, Qiang, OE

Yuan, Shengfu, 17

Yue, Jianbao, 12

Zhan, Kaiyun, OY

Zhang, Fan, OM

Zhang, Hai-kun, 02

Zhang, Jiabo, $1 \mathrm{~K}$

Zhang, Kai, 09

Zhang, Kaihu, $1 \mathrm{~K}$

Zhang, Kouli, $O Q$

Zhang, Liu-gang, 16

Zhang, Mingji, 10

Zhang, Mingqian, OJ

Zhang, Tianyun, 14

Zhang, Wenying, OL

Zhang, Ya-ping, 02

Zhang, Yongxiang, OL

Zhang, Yuxuan, OF, 1J

Zhao, Da-xing, 16

Zhao, Heng, 12

Zhao, Shubin, OT

Zheng, Jiangang, $\mathrm{OM}$

Zheng, Kuixing, OM

Zheng, Liyan, $1 \mathrm{~K}$

Zheng, Wanguo, OM

Zheng, Xiaoping, 14

Zhi, Dong, $O Q$

Zhou, Cheng, 02

Zhou, Kun, OX

Zhou, Pu, 09, 0J, 15, 19

Zhu, Qihua, OM

Zhu, Rihong, 06

Zhu, Yining, $1 \mathrm{~F}$ 


\title{
Conference Committees
}

\author{
Conference Chairs
}

Guangjun Zhang, Southeast University (China)

Byoungho Lee, Seoul National University (Korea, Republic of)

John Greivenkamp, The University of Arizona (United States)

Conference Review Committee

Desheng Jiang, Wuhan University of Technology (China)

Dianyuan Fan, Shenzhen University (China)

Huilin Jiang, Changchun University of Science and Technology (China)

Junhao Chu, Shanghai Institute of Technical Physics, Chinese Academy of Sciences (China)

Jianyu Wang, Chinese Academy of Sciences, Shanghai Branch (China)

Jianquan Yao, Tianjin University (China)

Jiancheng Fang, Beihang University (China)

Jiubin Tan, Harbin Institute of Technology (China)

Lin Li, University of Manchester (United Kingdom)

Lijun Wang, Changchun Institute of Optics, Fine Mechanics and Physics, Chinese Academy of Sciences (China)

Min Gu, University of Shanghai for Science and Technology (China)

Qionghai Dai, Tsinghua University (China)

Shibin Jiang, AdValue Photonics, Inc. (United States)

Shenggang Liu, University of Electronic Science and Technology of China (China)

Shouhuan Zhou, North China Research Institute of Electro-Optics (China)

Toyohiko Yatagai, Utsunomiya University (Japan)

Wenqing Liu, Anhui Institute of Optics and Fine Mechanics, Chinese Academy of Sciences (China)

Wei Wang, Beijing Institute of Aerospace Control Device of CASC (China)

Yueguang Lv, Chinese Academy of Engineering (China)

Zuyan Xu, The Technical Institute of Physics and Chemistry of the Chinese Academy of Sciences (China)

Conference Program Committee

Chaoyang Lu, University of Science and Technology of China (China)

Daoxin Dai, Zhejiang University (China)

Dong Liu, Zhejiang University (China)

Haimei Gong, Shanghai Institute of Technical Physics, Chinese Academy of Sciences (China)

Hongbo Sun, Tsinghua University (China)

Jin Lu, Tianjin Jinhang Institute of Technical Physics (China)

Jinxue Wang, SPIE 
Jin Yu, Shanghai Jiao Tong University (China)

Jian Zhang, Shanghai Institute of Ceramics, Chinese Academy of Sciences (China)

Min Gu, University of Shanghai for Science and Technology (China)

Min Qiu, Westlake University (China)

Michelle Sander, Boston University (United States)

Mengxia Xie, Beijing Normal University (China)

Pu Zhou, National University of Defense Technology (China)

Sven Hofling, Universität Wurzburg (Germany)

Shibin Jiang, AdValue Photonics, Inc. (United States)

Suijian Xue, National Astronomical Observatories, Chinese Academy of Sciences (China)

Takunori Taira, RIKEN SPring-8 Center (Japan)

Vincenzo Palleschi, Institute of Chemistry of Organic Metallic Compounds (Italy)

Wenxue Li, East China Normal University (China)

Xiaocong Yuan, Shenzhen University (China)

Xuejun Zhang, Changchun Institute of Optics, Fine Mechanics and

Physics, Chinese Academy of Sciences (China)

Yadong Jiang, University of Electronic Science and Technology of China (China)

Yongtian Wang, Beijing Institute of Technology (China)

Yuegang Fu, Changchun University of Science and Technology (China)

Zhiping Zhou, Peking University (China)

Zhe Wang, Tsinghua University (China)

Ziyang Zhang, Westlake University (China)

Session Chairs

1 Solid State Laser Technology and Applications

Takunori Taira, Institute for Molecular Science (Japan)

2 Advance Laser Technology

Minglie Hu, Tianjin University (China)

3 Ultrafast Laser

Dingyuan Tang, Jiangsu Normal University (China)

$4 \quad$ Mid-Infrared Laser

Jian Zhang, Shanghai Institute of Ceramics, Chinese Academy of Sciences (China)

$5 \quad$ Fiber Lasers

Pu Zhou, National University of Defense Technology (China)

6 Laser Materials

Deyuan Shen, Jiangsu Normal University (China) 


\section{Introduction}

AOPC 2019, the Annual Conference of Chinese Society of Optical Engineering (CSOE), was one of the largest academic and industrialization activities in the field of optical and optoelectronic technology in China. The Organizing Committee intended to build a potential platform together with academic exchanges, industry exhibitions, and cooperation negotiations in one. There were 11 technical conferences and 38 conference sessions, and we sincerely hope that the research and development of optoelectronic technology were promoted, and that international cooperation in industry and in the optical and optoelectronic fields were enhanced.

AOPC 2019 was sponsored by SPIE and the Chinese Society for Optical Engineering; the Optical Society of Korea (OSK), Optics and Photonics Society of Singapore (OPSS), European Optical Society (EOS), Optical Society of Japan (OSJ) were the technical co-sponsors. There were also 48 Cooperative Organizations that supported the conference.

We received over 930 total contributions from more than 15 countries, including United States, United Kingdom, Germany, France, Spain, Australia, Canada, Mexico, Brazil, Japan, the Republic of Korea, Thailand, Singapore, the Russian Federation, China, and so on. There were 554 contributions published in SPIE Proceedings.

After careful discussion, we suggested four keynote speeches which were presented by famous scientists from Germany, Australia, Japan, and China. There were 110 excellent invited talks, 45 of them are from overseas, their inclusion represented a first-class level in the field of optics and photonics technology. On behalf of the Organizing Committee of AOPC, I express the thanks to all the invited speakers and authors for their contributions and supports to the conference.

Finally, on behalf of the Chairmen and the Organizing Committee of AOPC, I would like to heartily thank for our sponsors and Cooperating Organizations for all they have done for the conference, to all of the participants and friends for their interests and efforts in helping us to make the conference success. Also, thanks to the Program Committee for their effective work and valuable advice; especially the AOPC 2019 Secretariat and SPIE for their tireless effort and outstanding services in preparing the conference and publishing the Proceedings.

We are glad that AOPC 2019 had great success! Hope to see you next year!

Guofan Jin 
Proc. of SPIE Vol. 11333 1133301-12

Downloaded From: https://www.spiedigitallibrary.org/conference-proceedings-of-spie on 26 Apr 2023 Terms of Use: https://www.spiedigitallibrary.org/terms-of-use 